TITLE:

\title{
Effect of oil droplet size on activation energy for coalescence of oil droplets in an $\mathrm{O} / \mathrm{W}$ emulsion.
}

\section{$\operatorname{AUTHOR(S):~}$}

Miyagawa, Yayoi; Katsuki, Kazutaka; Matsuno, Ryuichi; Adachi, Shuji

\section{CITATION:}

Miyagawa, Yayoi ... [et al]. Effect of oil droplet size on activation energy for coalescence of oil droplets in an O/W emulsion.. Bioscience, biotechnology, and biochemistry 2015, 79(10): 1695-1697

\section{ISSUE DATE:}

2015

URL:

http://hdl.handle.net/2433/202823

\section{RIGHT:}

This is an Accepted Manuscript of an article published by Taylor \& Francis in 'Bioscience, Biotechnology, and Biochemistry' on 12 May 2015, available online: http://www.tandfonline.com/10.1080/09168451.2015.1039482;; The fulltext file will be made open to the public on 12 May 2016 in accordance with publisher's 'Terms and Conditions for SelfArchiving'.; This is not the published version. Please cite only the published version.; この論文は出版社版でありません 。引用の際には出版社版をご確認ご利用ください。 
Running title: Activation energy for coalescence of oil droplets

Note

Effect of Oil-droplet Size on Activation Energy for Coalescence of Oil Droplets in an O/W Emulsion

Yayoi MIYAGAWA, ${ }^{1}$ Kazutaka KATSUKI, ${ }^{1}$ Ryuichi MATSUNO, ${ }^{2}$ and Shuji ADACHI ${ }^{1, \dagger}$

${ }^{1}$ Division of Food Science and Biotechnology, Graduate School of Agriculture, Kyoto University, Sakyo-ku, Kyoto 606-8502, Japan

${ }^{2}$ Professor Emeritus of Ishikawa Prefectural University and Kyoto University

Received February 25, 2015; Accepted March 24, 2015

$\dagger$ To whom correspondence should be addressed. Tel.: +81-75-753-6286; Fax: +81-75-753-6285; E-mail: adachi@kais.kyoto-u.ac.jp

The activation energy of a reasonable order of magnitude was estimated for the coalescence of oil droplets in an $\mathrm{O} / \mathrm{W}$ emulsion by formulating the balance of forces acting on a droplet that crosses over the potential barrier to coalesce with another droplet by the DLVO theory and Stokes' law. An emulsion with smaller oil-droplets was shown to be more stable.

Key words: O/W emulsion; dispersion stability; DLVO theory; stability factor; activation energy of coalescence

Oil-in-water $(\mathrm{O} / \mathrm{W})$ and water-in-oil $(\mathrm{W} / \mathrm{O})$ emulsions have been widely used in the food, cosmetic, and pharmaceutical industries. ${ }^{1)}$ The dispersion stability of emulsions, which are thermodynamically un- or quasi-stable, is very important from the viewpoint of their practical use. An $\mathrm{O} / \mathrm{W}$ emulsion with smaller oil droplets is empirically known to be more stable. Technologies have been developed for reducing the droplet size of the dispersion phase, and it has become possible to prepare an $\mathrm{O} / \mathrm{W}$ emulsion having oil droplets with a diameter of 100 nm or less. ${ }^{2)}$

The effects of the factors affecting the dispersion stability of the emulsion, such as the surface potential of the dispersion droplet and ion strength of the continuous phase, can be reasonably assessed based on the DLVO theory. ${ }^{3)}$ However, the DLVO theory predicts that the potential barrier between two adjacent droplets is lower for smaller droplets and that an emulsion with smaller droplets is more unstable. This prediction is inconsistent with experience. We have proposed a model to overcome the inconsistency for a dense $\mathrm{O} / \mathrm{W}$ emulsion, where an oil droplet to be coalesced with another oil droplet was thought to meet with frictional resistance from the continuous phase during coalescence; the frictional force was estimated by Stokes' law. ${ }^{4)}$ In the model, the balance of forces acting on a droplet was considered based on the DLVO theory and Stokes' law. The model successfully predicted that smaller oil droplets required larger kinetic energy to coalesce with another droplet; in other 
words, the smaller droplet was more stable. In this study, the model has been reconsidered to estimate the activation energy for the coalescence of oil droplets in a dense $\mathrm{O} / \mathrm{W}$ emulsion, and the effect of the oil-droplet size on the dispersion stability is examined for oil-droplet sizes ranging from $30 \mathrm{~nm}$ to $20 \mu \mathrm{m}$. The reconsidered model is called a hydrodynamic model.

The stability factor ${ }^{3,5)}$ derived by the diffusion model has been used to assess the dispersion stability. This factor was also evaluated under the same conditions as for the proposed model. The effect of the oil-droplet size on the dispersion stability, which was estimated based on this factor, was compared with the effect estimated by the hydrodynamic model.

Let us consider that two oil droplets with the same diameter, $d$, approach each other in a continuous phase. The surface potentials, $\psi_{0}$, of the droplets are also assumed to be identical. A droplet can approach point $x_{2}$, which is a far one of two points where the repulsive force is balanced by an attractive force, by diffusion and the density difference between the droplet and the continuous phase. Then, the droplet travels toward a near point $x_{1}$. The balance of forces acting on a droplet that crosses over the potential barrier to coalesce with another droplet is given by Eq. (1) by estimating the frictional force by Stokes' law.

$$
\frac{\pi}{6} d^{3} \rho_{\mathrm{s}} \frac{d u}{d t}=\frac{1}{4} \varepsilon d \psi_{0}^{2} \frac{\kappa \exp (-\kappa x)}{1+\exp (-\kappa x)}-F_{\mathrm{a}}-3 \pi d \eta u
$$

where $t$ is the time, $u(=d x / d t)$ is the velocity of a droplet, $x$ is the distance between the droplet surfaces, $\varepsilon$ is the dielectric constant of the continuous phase, $\eta$ is the viscosity of the continuous phase, $\kappa$ is Debye's factor, and $\rho_{\mathrm{s}}$ is the density of the droplet. The first, second and third terms of the right-hand side of Eq. (1) represent electrostatic repulsion, interparticle attraction and frictional resistance, respectively. In the previous model, ${ }^{4)}$ the attractive force $F_{\text {a }}$ was expressed in the approximate form, but Hamaker's strict solution for the force ${ }^{6)}$ is used in this study, as given by Eq. (2).

$$
F_{\mathrm{a}}=\frac{A}{24 d}(\chi+1)\left[\frac{1}{\left(\chi^{2}+2 \chi\right)^{2}}+\frac{1}{\left(\chi^{2}+2 \chi+1\right)^{2}}-\frac{2}{\chi^{2}+2 \chi}+\frac{2}{\chi^{2}+2 \chi+1}\right]
$$

where $\chi=x / d$ and $A$ is Hamaker's constant.

In the previous study, ${ }^{4}$ it was considered as the initial conditions for Eq. (1) that a droplet located at point $x_{2}$ gains $a$ times the kinetic energy of a molecule $k T$, where $k$ is Boltzmann's constant and $T$ is the absolute temperature, and this droplet begins to move toward another droplet. The multiple $a$ is apparently dimensionless, but it has units of the number of molecules per number of droplets. To estimate the effect of the type of lipid molecule consisting of the droplet on the dispersion stability and to estimate the rate constant for coalescence, the activation energy, $E$, for coalescence was used as an index of the dispersion stability.

When an oil droplet with diameter $d$ consists of a lipid with molecular mass $M$, the droplet contains lipid molecules of $(\pi / 6) d^{3} \rho_{\mathrm{s}} / M$ mol, which can be converted to the number of lipid molecules by multiplying the number of moles by the Avogadro number $N_{\mathrm{A}}$. Let us consider the situation in which all lipid molecules consisting of the oil droplet simultaneously gain the kinetic energy of a molecule, $k T$, in the direction to migrate toward another droplet. Under this condition, the initial conditions for Eq. (1) are given by the following equation: 


$$
t=0, x=x_{2} ; \quad \frac{\pi}{12} d^{3} \rho_{\mathrm{s}} u^{2}=a_{0} \frac{(\pi / 6) d^{3} \rho_{\mathrm{s}}}{M} k T N_{A}=a_{0} \frac{(\pi / 6) d^{3} \rho_{\mathrm{s}}}{M} R T
$$

where $R$ is the gas constant and $a_{0}$ is the multiple of the minimum kinetic energy for the droplet to cross over the potential barrier to coalesce with another droplet. $a_{0}$ can be converted to the activation energy, $E$, by Eq. (4).

$$
E=a_{0} k T N_{\mathrm{A}}=a_{0} R T
$$

The activation energy, $E$, of the droplet with diameter $d$ was calculated using the Runge-Kutta-Gill method under the conditions of $A=10^{-20} \mathrm{~J}, T=298 \mathrm{~K}, \varepsilon=6.90 \times 10^{-10} \mathrm{~F} / \mathrm{m}$, $\eta=5.0 \times 10^{-3} \mathrm{~kg} / \mathrm{m} \cdot \mathrm{s}, \kappa=1.0 \times 10^{-8} \mathrm{~m}^{-1}, \rho_{\mathrm{s}}=888 \mathrm{~kg} / \mathrm{m}^{3}$, and $\psi_{0}=5.0 \times 10^{-2} \mathrm{~V}$, which are the same as those used in the previous report. ${ }^{4)}$ The oil droplet was assumed to consist of ethyl oleate with molecular mass of $0.310 \mathrm{~kg} / \mathrm{mol}$. The solid curve in Fig. 1 shows the relationship between the $E$ and $d$ values. The $E$ value was larger for the smaller oil droplet, indicating that the emulsion with smaller oil droplets is more stable. The $E$ value for the emulsion having oil droplet smaller than $1 \mu \mathrm{m}$ was roughly in the range of 10 to $10^{3} \mathrm{~kJ} / \mathrm{mol}$, which are almost the same as the values for many physical and chemical processes.

The collision frequencies of oil droplets with and without the potential barrier are denoted as $G$ and $G_{V=0}$, respectively. The stability factor, $W$, is defined as the ratio of $G_{V=0}$ to $G$, and it can be calculated by Eq. (5). ${ }^{3)}$

$$
W=\frac{G_{V=0}}{G}=d \int_{d}^{\infty} \frac{e^{V / k T}}{r^{2}} d r
$$

where $r$ is the distance from the center of the fixed oil droplet, and $V$ is the potential barrier, which is given by Eq. (6).

$$
V=\frac{1}{4} \varepsilon d \psi_{0}^{2} \ln [1+\exp (-\kappa x)]-\frac{A}{12}\left(\frac{1}{\chi^{2}+2 \chi}+\frac{1}{\chi^{2}+2 \chi+1}+2 \ln \frac{\chi^{2}+2 \chi}{\chi^{2}+2 \chi+1}\right)
$$

The $W$ values were numerically calculated using the Simpson's rule at various $d$ values (ranging from $30 \mathrm{~nm}$ to $20 \mu \mathrm{m}$ ) under the same conditions as for the hydrodynamic model, as shown by the broken curve in Fig. 1. The stability factor was the largest for a droplet of $\sim 3-\mu \mathrm{m}$ diameter, and it sharply decreased for droplets with smaller diameters. Verwey and Overbeek $^{3)}$ also calculated the dependency of the stability factor on the diameter of the dispersion phase, and they showed that the stability factor was maximized at a dispersion phase of $100 \mathrm{~nm}$. The difference in the diameter of the dispersion phase between our and their results is attributed to the difference in the parameters used. However, the tendency that the stability factor increased to a maximum value and then declined sharply as the diameter of the dispersion phase decreased was common in both results. This tendency is inconsistent with the oil-droplet size dependency of a practical emulsion, where the dispersion stability is higher for smaller oil droplets. For example, commercial milk is homogenized to reduce the oil-droplet size to $\sim 500 \mathrm{~nm}$ to improve the dispersion stability.

The hydrodynamic model successfully indicated that an emulsion with smaller oil droplets was more stable by incorporating the frictional force with the DLVO theory. Spielman ${ }^{7)}$ and Vinuesa $e t a l .{ }^{8)}$ discussed the stability factor by considering the frictional force and suggested the importance of the effect of the frictional factor on the dispersion stability. Therefore, the frictional force should play an important role in the dispersion stability of emulsions. 
Author contribution

R.M. and S.A. designed the research. Y.M. and K.K. performed the calculations. Y.M. and S.A wrote the article discussing with R.M. and K.K.

\section{References}

[1] Chappat M. Some applications of emulsions. Colloids Surf. A 1994; 91: 57-77.

[2] Jafari SM, Assadpoor E, He Y, Bhandari B. Re-coalescence of emulsion droplets during high-energy emulsification. Food Hydrocolloids 2008; 22: 1191-1202.

[3] Verwey EJW, Overbeek JThG. Theory of the stability of lypophobic colloids, Amsterdam: Elsevier; 1948.

[4] Adachi S, Imagi J, Matsuno R. Model for estimation of the stability of emulsions in a cream layer. Biosci. Biotech. Biochem. 1992; 56: 495-498.

[5] Fuchs N. Über die Stabilität und Aufladung der Aerosole. Z. Physik 1934; 89: 736-743.

[6] Hamaker HC. The London-van der Waals attraction between spherical particles. Physica 1937; 4: 1058-1072.

[7] Spielman LA. Viscous interactions in Brownian coagulation. J. Colloid Interf. Sci. 1970; 33: 562-571.

[8] Vinuesa JLO, Rodríguez AM, Álvarea RH. Colloidal stability of polymer colloids with different interfacial properties: Mechanisms. J. Colloid Interf. Sci. 1996; 184: 259-267.

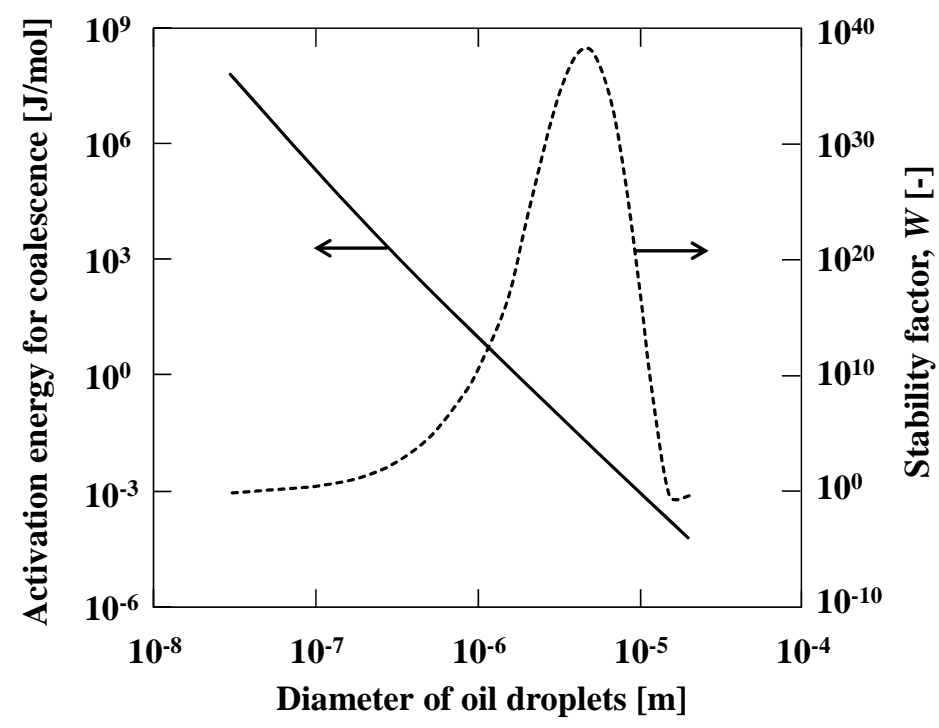

Fig. 1. Effect of oil-droplet size on activation energy (solid curve) and stability factor (broken curve). 